\title{
PULSED MAGNETIC FIELD MEASUREMENT USING A FERRITE WAVEGUIDE IN A PHASE BRIDGE CIRCUIT
}

\author{
W. Pellico,P. Colestock, Fermi National Accelerator Laboratory ${ }^{\dagger}$, P.O. Box 500, Batavia, Ill., USA, \\ 60510 \\ FERMILAB-Conf-97/437 July 2002
}

\begin{abstract}
There are several standard methods used for measuring pulsed magnetic fields. However the induction or Hall probe methods have limited bandwidth and experience reflection problems. The integrated magnetic field can only be found by measuring along the entire length of the magnet. Problems with reflections, noise and bandwidth will limit the accuracy of measurement. Presented in the following paper is a method for measuring pulsed fields without the typical noise errors and bandwidth limitations. This paper will describe a phase bridge network that relies upon the permeability of a ferrite waveguide to accurately measure the integrated field of a Main Injector kicker magnet. We present some data taken with the system, a first pass at the analysis of this data, and discuss some possible design variations.
\end{abstract}

\section{INTRODUCTION}

In pulsed magnetic systems, the measurement the integrated magnetic field is a difficult process, which usually results in limited accuracy. One method of ascertaining the integrated magnet field is by taking current probe data from both ends of the kicker and integrating. Another method is to use a shielded coil loop to measure a changing magnetic field. Both methods will result in limited accuracy due to the large electric field present in kickers, reflections over the probe length and bandwidth limitations of the measuring device. These problems can be eliminated (or greatly reduced) if the probe's susceptivity to electric fields and signal reflections along the conductor are reduced. The choice of ferrite with high intrinsic impedance makes it an ideal probe for pulsed field measurement.

\subsection{Phase Bridge System}

The ferrite probe is actually a waveguide, which acts as one of the legs in a phase bridge circuit. A radio frequency (RF) signal is split down both legs of the circuit. One of the signals is coupled into the ferrite guide and the other is sent into a delay line. The ferrite is placed inside the center of the kicker aperture. The emerging ferrite guide is then coupled back into cable and mixed with the delay line signal. The DC mixing term is

\footnotetext{
${ }^{\dagger}$ Operated by the Universities Research Association, Inc. under contract with the U.S. Department of Energy.
}

then low passed before being measured on a fast digitizing oscilloscope. In the absence of an externally applied magnetization, the phase bridge is measured and balanced. When the magnetization pulse occurs a phase unbalance develops between the two legs of the phase bridge. The unbalance is a result of the magnetization of the ferrite, which changes the wave velocity of the guided RF signal.

\subsection{System Specification}

The quality of the measurement will depend largely upon choice of ferrite. There are numerous types of ferrite and each have material characteristics that need to be matched for the particular pulsed magnetic system. The ferrite(s) used in this testing was based upon the specifications of the Main Injector kicker. Since the shifting of the wavelet velocity will result is a phase shift, the greater the phase shift the larger the resulting mixed DC component.

Another key factor is the frequency of the signal used in the system. The higher the frequency, the larger the number of waves inside the ferrite which results in higher sensitivity. The RF will also determine the bandwidth of the measurement system and should be well above the highest frequency component in the pulsed magnet waveform. The magnetization pulse was calculated to have a frequency spectrum of DC to 100 $\mathrm{MHz}$. The high frequency is also a requirement for single-mode operation in a ferrite guide, which must have small cross-sectional dimensions. Listed below are several of the kicker system specifications, which were used in determining the choice of ferrite and RF:

\begin{tabular}{|c|c|}
\hline $\begin{array}{ll}\text { - } & \text { Nominal Field } \\
\text { - } & \text { Magnetic Length } \\
\text { - } & \text { Field Rise Time } \\
\text { - } & \text { Aperture H.V }\end{array}$ & $\begin{array}{l}136 \text { gauss } \\
0.83 \text { meters } \\
-50 \text { nsec. } \\
1600 \mathrm{nsec} \\
101.6 \mathrm{~mm} \times 50.8 \mathrm{~mm}\end{array}$ \\
\hline
\end{tabular}

The frequency chosen for our measurement was an $\mathrm{X}$-band (9 to $10 \mathrm{GHz}$ ) signal. Two ferrites which complied with the requirements and used in the system are G-1009 and G-250, both are manufactured by TransTech, Inc. The table below lists several of the two ferrite materials characteristics. The data comes from a 1992 Trans-Tech microwave materials data book. 
- FERRITE

- Saturation M

- Permeability

- Dielectric

The choice of mixer was determined by its passband flatness and expected power levels Choice of components were also based upon availability and cost.

\section{LAYOUT}

The phase bridge system is shown in figure 1 . The RF source was a network analyzer with an output power of $+12 \mathrm{dBm}$ (A $150 \mathrm{MW} 10 \mathrm{GHz}$ Gunn source was also tried.) The signal launched into the waveguide was approximately $+8 \mathrm{dBm}$. The waveguide to ferrite coupler was a machined Aluminum block with quarter wave steps on the input end and a flared output on the output end for impedance matching. After passing through the kicker, the ferrite was then again coupled back into coax. Both upstream and downstream launches were identical. A waveguide isolator was attached at both ends of the launch. The upstream waveguide section also had a waveguide phase shifter attached. This allowed for about 400 degrees of phase tuning. The phase shifted signal was then mixed with the delayed line RF signal and then low passed filtered. We did use an IF amplifier in some of the test runs but found it wasn't necessary in all cases. The low pass filter cutoff of $200 \mathrm{MHz}$ was well above the harmonic content of the magnetic field pulse but still eliminated noise spikes and other higher order terms of the mixing. The $\mathrm{DC}$ to $100 \mathrm{MHz}$ signal was then sampled on a fast oscilloscope and saved to disk for analysis. The scope needed to be housed in a shielded cage for EMI reduction (not shown in figure 1).

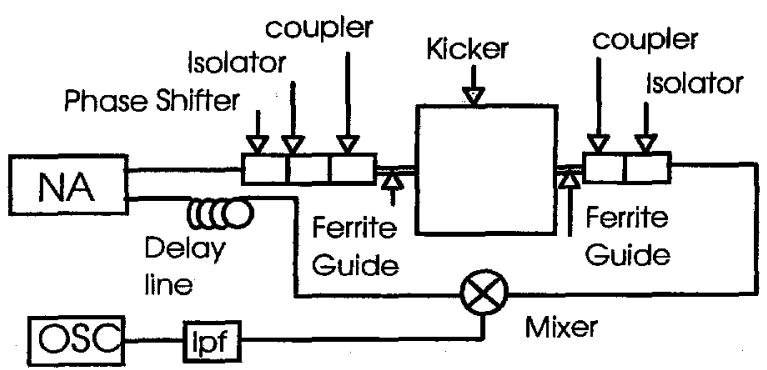

Figure 1:Phase Bridge Measurent Setup

\section{MEASUREMENTS}

Measurements of the pulsed kicker system were taken over a two week period. Small modifications to the measurement system continued throughout the testing period. Prior to using the ferrite probe system, a printed circuit field coil and capacitive pickup probes were used to measure the pulsed magnetic field [1]. Although those measurements were affected by some noise and ringing,

the results can give a fairly accurate magnetic field profile against which to compare the ferrite probe data.

The phase bridge measurements were taken with various kicker voltage and reservoir settings. The tests were to confirm the performance of the phase bridge system as well as the pulse magnet.

\subsection{Flattop Measurement}

A critical aspect of kicker systems is the flattop stability. We took two sets of flattop data. The first set was a measurement of the flattop field verses various kicker voltage settings to see if the measured magnetic field scaled properly (see figure 2.) The second data set measured the kicker flattop when the impedance matching of the kicker and its termination load was being changed. The idea is to measure the flattop when the kicker is terminated in a matched load to that of a slightly different load value. This was done by heating the load with a higher pulse rate.

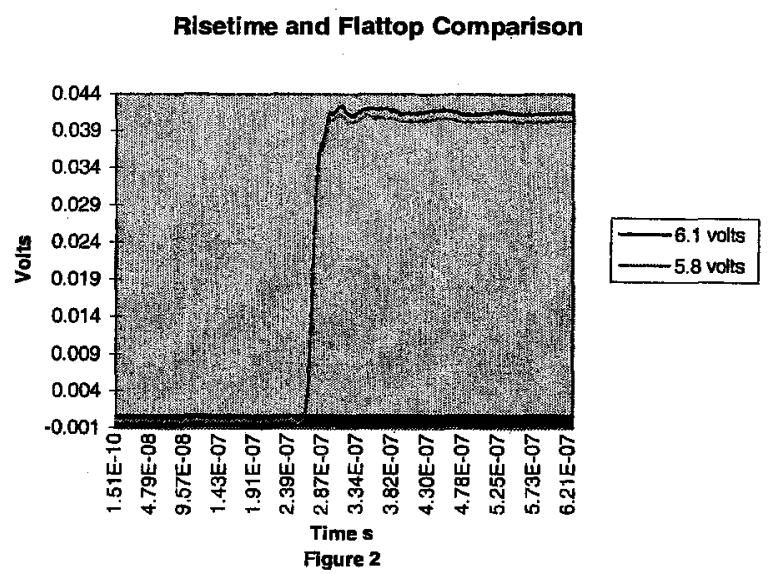

The data from the first set of measurements shows an ability to measure voltage changes to better than $2 \%$. This is more a test of relative change rather then a measure of absolute field value. Since the only way to get absolute values is to DC test the ferrite and trace out the characteristic B-H curve for the given ferrite waveguide. This test is being done at the writing of this paper.

The second set of data shows evidence of a kicker mismatch. The flattop has developed a small perturbation with a period related to the load and kicker length. The impedance mismatch is not accurate enough to be used as a calibration. But the flattop measurement was able to detect a $3 \%$ change in load resistance (see figure 3 .) 


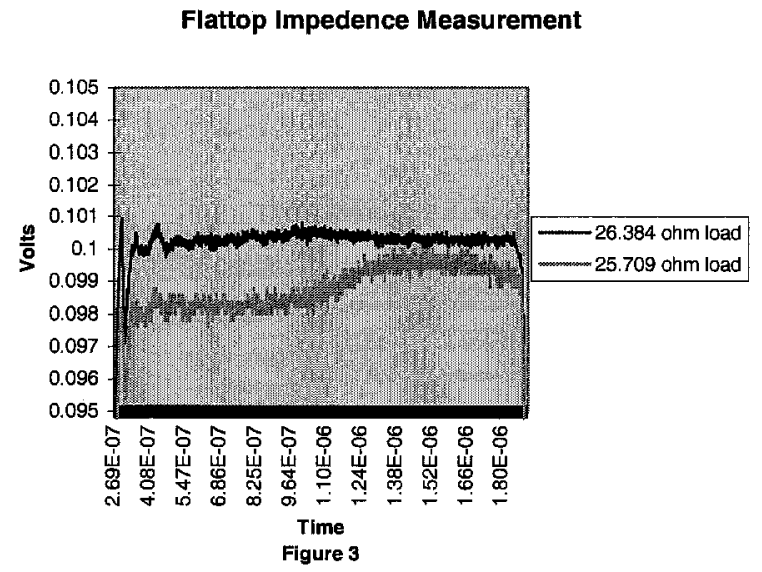

\subsection{Risetime Measurements}

Another critical aspect in kicker performance is the magnetic field risetime. The risetime was measured from base line to when the integrated field has reached flattop. The Main Injector kicker is specified to have a 50 ns risetime.

We measured the risetimes as a function of kicker voltage settings and at various tube gas pressures. The phase bridge circuit measured a risetime at nominal kicker settings of approximately 30ns. The comparison with that of a current probe showed a $12 \mathrm{~ns}$ faster risetime. This difference can be partly explained by the systemic error of the two systems. The current probe is coupled through a $\sim 30$ pf capacitor and shows signs of being critically damped. This reduction in bandwidth could result in a longer risetime.

\section{SYSTEM DESIGN ALTERNATIVES}

We used a network analyzer as a source. A high -loss ferrite may require an additional amplifier. An IF amplifier could be attached before the mixer. Another option is to mix down the $\mathrm{X}$ band signal before passing through a log amplifier. The $\log$ amplifier will reduce errors associated with amplitude variations.

\section{CONCLUSION}

Preliminary field measurement data shows the possibility of using a ferrite rod as a waveguide in a phase bridge setup. The accuracy of the field measurement will be proven with beam tests when the Main Injector comes on line. The agreement with standard measurement techniques shows that the phase bridge system is a viable measurement. Used in conjunction with standard methods, a more accurate magnetic field profile is obtained. More testing will need to be done to improve upon the accuracy, but the present system can deliver 1 to $2 \%$ accuracy.

\section{ACKNOWLEDGMENTS}

The authors acknowledge Bruce Hanna for his assistance in the project. We would also like to thank Peter Bagley

\section{REFERENCE}

[1] D. Quenell, "Kicker System for $8 \mathrm{Gev}$ Proton Injection", PAC 97 , These Proceedings 